\title{
The Effects of Increasing Antenna Arrays for MIMO in Mine Tunnels
}

\author{
Ali Nehme $^{1}$, Nadir Hakem ${ }^{1}$, and Nahi Kandil ${ }^{1}$ \\ ${ }^{1}$ LRTCS-UQAT, 450, 3eme Avenue Local 105, Val-d'Or, Québec, J9P 1S2, Canada
}

\begin{abstract}
The aim of this paper is to prove theoretically by using waveguide and geometrical optical models that increasing MIMO array elements at the transmitter and receiver will have a limit on capacity where the equivalent spatial subchannels can be limited by the number of allowable modes.
\end{abstract}

\section{Introduction}

In confined environments such as tunnels and mines, high communication data is necessary for various subjects like ensuring safety by providing communication between workers anywhere, anytime. Recently, MIMO had been embraced by the underground mining community, which has received unprecedented attention due to its reliability and capacity compared to single antenna transmission. However, MIMO can be more complicated compared to SISO especially in waveguide like environments. In the free space environment, the channels are assumed to be uncorrelated and that because of the rich scattering environment, degradation of the link performance can occur whenever we have a correlation between fading of each channel, and that is the case in mine tunnel where we have a poor scattering environment. Before deploying the MIMO system, the MIMO channel should be accurately characterized. In small mine tunnels which have a narrow width, it's not effective to deploy high MIMO antenna arrays, and that because of some theoretical and practical aspects such as channels correlation, the actual number of modes contributing to the power at the received signal and the antenna array configuration [1]. In our study, we consider that mines can act as tunnels in case of comparing them to over-sized dielectric waveguide where many modes can propagate [2], and this if we consider embracing the waveguide model, but the problem with the latter that it's not efficient in the near field region, where the geometrical optical model (GO) can numerically solve the problem of the near field region by predicting the paths [3]. Correspondingly, work done in [4] where a new hybrid model is obtained, the "multi-mode model", where it combines the two models. However, instead of using single antennas, MIMO is considered in this work, since Maxwell equations can solve the modes of the waveguide model. Hence, modal expansion theory can be used in tunnels and that after a successful study in [5] and [6], and that by considering the first modes as "quasi-orthogonal".
In this work, we have conducted theoretical MIMO analysis in underground mines to interpret the effect of increasing antenna array elements on the system's capacity. The remainder of this paper is organized as follows. In Section II, a modal analysis in mine tunnels is introduced and we calculated the number of modes in the underground mine environment. In section III, we discussed the relation between MIMO antenna array size and the performance of the system and theoretical validation is done to verify our claim. Finally, the paper is concluded.

\section{Modal analysis of lossy waveguide environments}

The aim of this section is to determine the number of modes in an oversized lossy waveguide. Subsequently, the number of propagation channels can be determined. Underground mine walls are different from that in the ideal waveguides, where the existing of imperfect walls. The modal expansion theory of the fields is complicated by the coupling of the basic modes by the imperfect walls and this is due to the boundary conditions in the walls of the guide which raises a fundamental difficulty in obtaining the modal eigenvalues and eigenfunctions, and this is according to wait [7]. Indeed, high order modes are subject to high attenuation at large distances from the transmitter. Hence, according to [8], we can consider taking the first 60 modes as "quasi-orthogonal". In order to determine the number of modes, we applied the waveguide model to determine the mode intensities in the mine tunnel, while we used ray tracing to obtain the EM rays in the tunnel.

\subsection{Multi-Mode propagation in lossy waveguides}

Mine tunnels are separated into two regions, the near field, and the far field. In the cross-sectional plane where the transmitter is located, the antenna excites multiple modes where each one has different intensity and phase, 
the high order modes are considered to be evanescent modes at large distances and that because of the high attenuation factor they have, and this attenuation results from impinging the walls within a grazing angle. Contrarily, in the far field region, only the fundamental modes survive and they contribute to the total received power at the receiver. The separation breakpoint between these two fields is still hard to be obtained in a direct manner due to the different environment shapes.

\subsection{Theoretical approach for Determining the number of modes}

By solving the Maxwell's equations, the field distribution of each mode can be derived in the form of eigenfunctions as follows [5], [9]:

$$
E_{m, n}^{e i g n} \simeq \sin \left(\frac{m \pi}{2 a} x+\varphi_{x}\right) \cdot \cos \left(\frac{n \pi}{2 b} y+\varphi_{y}\right)
$$

Where $\varphi_{y}=\left\{\begin{array}{cl}0, & \text { if } n \text { is odd } \\ \frac{\pi}{2}, & \text { if } n \text { is even }\end{array}\right.$

$$
\text { and } \quad \varphi_{x}= \begin{cases}0, & \text { if } m \text { is even } \\ \frac{\pi}{2}, & \text { if } m \text { is odd }\end{cases}
$$

where Arbitrary electromagnetic field inside of such a waveguide can be expanded in terms of the eigenmodes [3], [10]:

$$
E_{x, y, z}=\sum_{n} \alpha_{n} e_{n}(x, y) e^{-j k_{z n} z}
$$

Where $e_{n}(x, y)$ is the normalized modal function of the electric fields which are represented in (1), and $\alpha$ is the mode amplitudes. It's important to estimate the number of modes in any waveguide environment, obviously for underground mines it's quite complicated especially when high order modes start losing their orthogonality. We can consider calculating the amplitudes of the first modes by knowing the $\mathrm{E}$ and $\mathrm{H}$ fields in each cross-section of the tunnel. Furthermore, the only modes available in the waveguide are those who can have the same shapes in the existing fields [4], the latter can be obtained by ray tracing model where the mode amplitudes can be obtained by correlating the E field to the mode functions at a certain cross-sectional area $Z_{R}$ as follows:

$$
\alpha=\iint_{S} \mathbf{E}\left(\mathrm{x}, \mathrm{y}, \mathrm{z}_{\mathrm{R}}\right) \mathbf{e}_{\mathbf{i}}(\mathrm{x}, \mathrm{y}) \mathrm{dx} d \mathrm{y}
$$

In order to obtain the proper number of propagating channels, we assume calculating only the modes which contributes to at least $10 \%$ of the total received power, in this way we will avoid taking the evanescent modes as a real propagating channel. For instance, determining the number of modes in an ideal rectangular waveguide can be much simpler, where the maximum number of modes existed in a certain cross-section can be calculated by knowing the cross-sectional area and the frequency as follows:

$$
N \approx \frac{2 \pi A_{r e c}}{\lambda^{2}}
$$

Where $A_{\text {rec }}$ is the area of the rectangular cross-section. Correspondingly, only the number of orthogonal modes can determine the degrees of freedom of the system [9]. According to (4), we can set the maximum degrees of freedom of a certain environment by considering taking the minimum number of orthogonal modes within the desired area of interest where the implantation of the wireless system will take place.

\subsection{System model and theoretical validation}

Wireless InSite's ray tracing capability has been used to set up a small underground old gold mine scenario located in Val d'Or, Canada. The imported mine's walls have some protrusions as shown in Fig 1. We set up two scenarios, which have the same height and length $(\mathrm{H}: 3.5$ $\mathrm{m}, \mathrm{L}: 400 \mathrm{~m}$ ) but with a different width (w1: $3.5 \mathrm{~m}, \mathrm{w} 2: 7$ $\mathrm{m})$ and named as Case I and Case II. The tunnel stretches over a length about $400 \mathrm{~m}$ and it's broken down into two parts with $200 \mathrm{~m}$ length each: a LOS part and a NLOS part as shown in Fig 2. The transmitting and receiving antennas used are dipoles which are vertically polarized and uses a wide raised-cosine pulse. Correspondingly, a 2 $\lambda$ separation distance must be taken between antennas to ensure decorrelation between the subchannels. Indeed, a larger separation will not necessarily work for this distance. In contrast, smaller separation will increase the correlation, a further explanation can be found in [11]. The carrier frequency used is $900 \mathrm{MHz}$. In Fig 2, we placed the transmitter array at point $\mathrm{A}$, and the receiver points are aligned along the trajectory from point $\mathrm{A}$ to $\mathrm{B}$ with a 1-meter separation distance. According to the previous experimental work in [11] and [12], it has been proved that aligning the antenna array perpendicular to the tunnel axis will perform better than other orientations. Hence, we adopt this orientation in our study. The configuration of ray tracing calculation is defined to use wireless InSite's GPU accelerated X3D ray model, the model can handle arbitrary geometry and transmitters/receivers at any height, this accurate model includes reflections, transmissions and diffractions along with frequency dependent atmospheric absorption. Besides, ray tracing defined to include propagation paths with up to six reflections and one diffraction omitting transmissions to limit calculation to the outdoors.

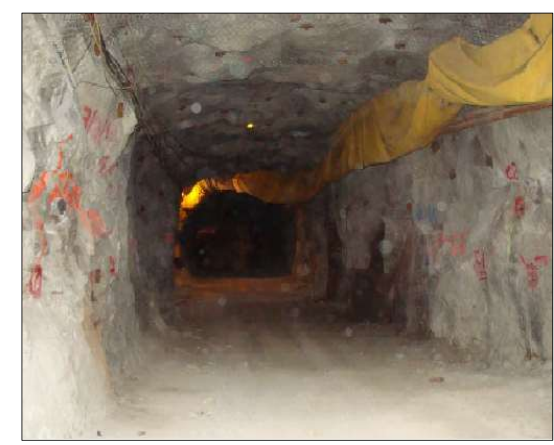

Fig. 1. CANMET mine image 


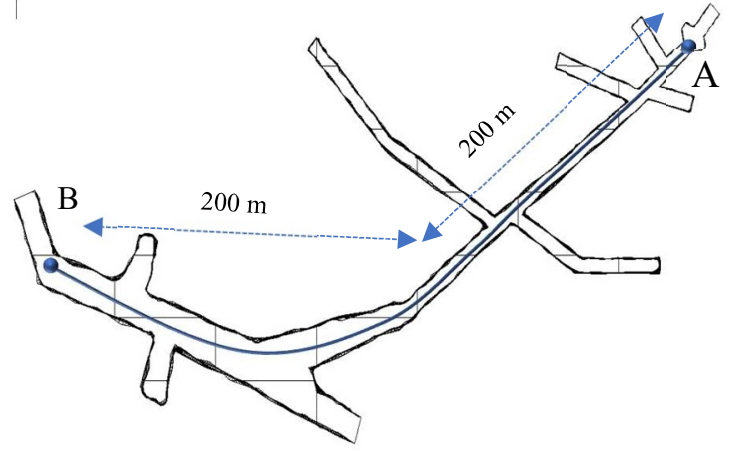

Fig. 2. CANMET Schematic.

\subsection{Results}

In Fig 3, we can interpret from the graph that the wider tunnel records a higher number of modes within the first $200 \mathrm{~m}$, and that's because in this region the tunnel's act as a free space environment where the lower angular spread in the narrowest tunnel causes a higher correlation between the arrays, which in terms causes deterioration in the received power. Contrarily, the coupling between excited modes at the transmitter may have a negligible effect on the performance in the far field region. Correspondingly, we can see that after $300 \mathrm{~m}$, the two tunnels record nearly the same number of modes.

\section{MIMO channel characteristics in underground mine}

In this section, we will prove that increasing the size of the MIMO antenna array in tunnels will not necessarily increase the performance of the wireless communication system. The environment and measurement procedures used here are the same as in section II, except that we used

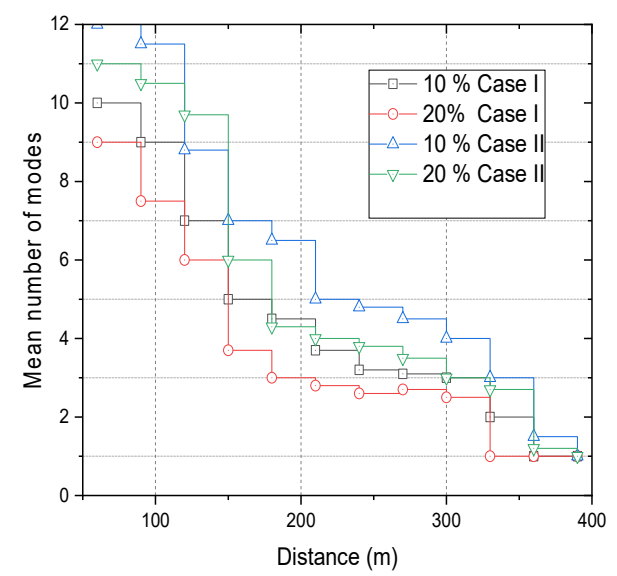

Fig. 3. Mean number of modes in CANMET for Case I and Case II.
Case I measurements. In addition, we considered taking $2 \times 2,4 \times 4$ and $6 \times 6 \mathrm{MIMO}$ antenna arrays to examine the power and capacity of each system.

\subsection{MIMO capacity}

If we consider taking $\mathrm{M} \times \mathrm{N}$ memoryless MIMO system, the maximum capacity without water-filling and in the presence of white Gaussian noise is given by Foschini et al.

$$
\mathrm{C}=\log _{2} \operatorname{det}\left(\mathrm{I}_{\mathrm{m}}+\sigma \cdot \mathrm{HH}^{\mathrm{H}}\right) \text { bits } / \mathrm{s} / \mathrm{Hz}
$$

Where $I_{m}$ represent the identity matrix; $\mathrm{H}$ is the channel matrix, and $\sigma$ represents the SNR of each antenna. The channel capacity can be expressed in terms of the singular values of the matrix as follows:

$$
\mathrm{C}=\sum \log _{2}\left(1+\frac{\mathrm{E}_{\mathrm{x}}}{\sigma^{2}} \lambda_{\mathrm{i}}\right)
$$

For $\mathrm{H}=\mathrm{I}$, and for identical number of antennas at the transmitter and receiver $\mathrm{N}$, we can represent MIMO capacity in (6) where the maximum capacity is achieved for independent subchannels as follows [9]:

$$
\mathrm{C}=\mathrm{N} \operatorname{det}(1+\sigma)
$$

From these equations, we can interpret that improving the system's capacity will undoubtedly depend on the number of antennas and the SNR. Hence, we assumed taking a constant SNR, thus we used Euclidean norm to normalize the complex H matrix. When constant SNR is assumed, it allows emphasizing the effect of channel change on the correlation and thus the change in path loss with distance is removed.

\subsection{Theoretical approach}

Low capacity can occur in MIMO even when we have zero correlation between the signals. This is the case of degenerated channels. For MIMO, the wireless channel can be represented as a matrix of the fading channels with additive noise.

$$
\bar{y}=\mathrm{H} \overline{\mathrm{x}}+\overline{\mathrm{w}}
$$

Where $\mathrm{y}$ is the received signal array, $\mathrm{x}$ is the transmitted signal and $\mathrm{w}$ is the additive white Gaussian noise. From Foschini et al MIMO capacity formula, we can realize that the channel capacity depends on the channel matrix $\mathrm{H}$. The fading channel $\mathrm{H}$ is decomposed by singular value decomposition (SVD), Correspondingly, the wireless system can be represented as follows:

$$
\overline{\mathrm{y}}=\mathrm{U} \Sigma \mathrm{V}^{\mathrm{H}} \overline{\mathrm{x}}+\overline{\mathrm{w}}
$$

after performing precoding to (9), the system now can be decomposed into many propagation channels. Indeed, as was proposed by Loyka, that individual waveguide modes can act as MIMO propagating channels. In an ideal waveguide, the modes are considered orthogonal if the 
mode coupling factor is represented in the following sense [loyka]:

$$
\iint \mathrm{e}_{\mathrm{n}} \mathrm{e}_{\mathrm{m}} d \mathrm{x} d \mathrm{y}=\delta_{m n}
$$

Where $e_{n}(x, y)$ is the normalized modal functions of the electric field. For mine tunnel environment, the orthogonality is still applied for the first 60 mods, where the mode coupling factor is considered to be neglected [3]. Accordingly, spatial multiplexing can be applied. Besides, if we have an $\mathrm{M} \times \mathrm{N}$ MIMO system we must have $L \geq \min (M, N)$ to profit from this technique. Where $\mathrm{L}$ is the number of active modes [8], [5].

\subsection{Results discussion}

As shown in Fig 5 , the power received by $4 \times 4$ and $6 \times 6$ MIMO systems is nearly the same, the reason is that in the near filled region, the excited modes forms about $60 \%$ of the total power contributed at the receiver, where the first 3 modes form only $5 \%$, the remaining power is distributed within the medium modes. In the near field region, the signal drops fast and we can analyze a big fluctuation wherein the far field region the fundamental modes now form $90 \%$ of the total power, obviously we can analyze a drop in the attenuation rate in the NLOS, the breakpoint can be analyzed visually after $130 \mathrm{~m}$ and that by analyzing a periodic signal. We can analyze that the performance of high orders MIMO are not as we can expect in the free space, because the fundamental modes which are orthogonal to each other are smaller than the propagation channels. Furthermore, if we consider taking higher frequencies, we expect to have a less attenuation drop but we don't expect improvement in the capacity with increasing the number of antennas.

In Fig 5 and Fig 6, we can analyze that the capacity of $2 \times 2$ and $4 \times 4$ MIMO systems are nearly the same and in sometimes they exceed the capacity of the $6 \times 6$ MIMO system, and this is the situation where the degrees of freedom of the system is reduced with distance and it became less than the available transmitting channels. We can expect that $6 \times 6 \mathrm{MIMO}$ can perform better in Case II

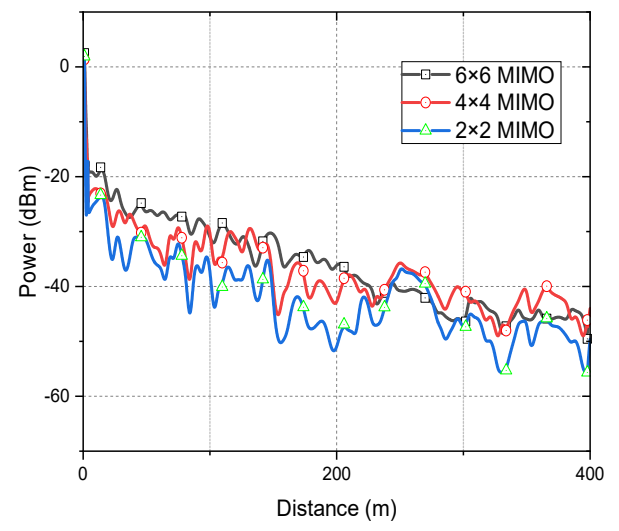

Fig. 4. Power versus distance between different MIMO systems.

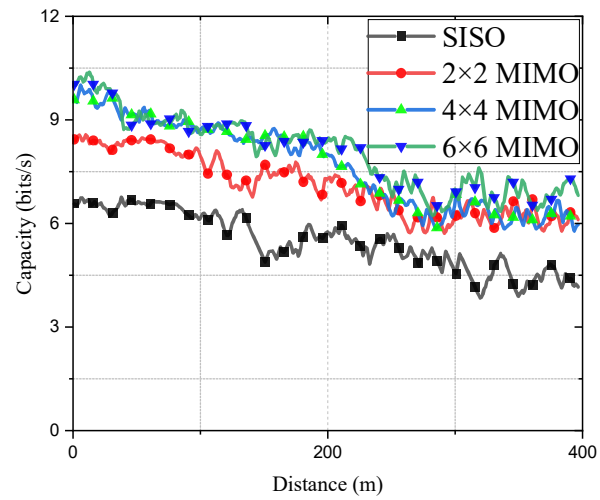

Fig. 5. Capacity versus distance between different MIMO systems.

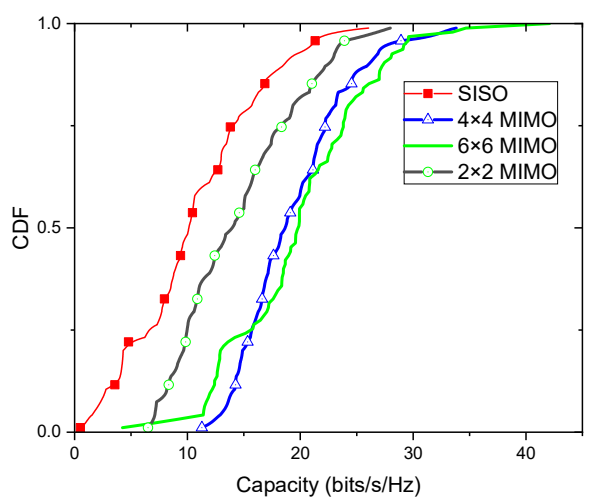

Fig. 6. CDF capacity between different MIMO systems

tunnel compared to $4 \times 4$ MIMO. In brief, if we have $\mathrm{M} \times \mathrm{N}$ MIMO system we must have $L \geq \min (M, N)$ to profit from this technique. Where $\mathrm{L}$ is the number of active modes [9] [12].

\section{Conclusion and future work}

MIMO capacity of mine tunnel has been discussed in this paper, a channel model which can calculate the EM field in the near and far field region is used to prove that in order to profit from the spatial multiplexing of the MIMO system we must have a number of antenna arrays which is equivalent to the number of modes at the receiver. Otherwise, MIMO improvement will be limited. Furthermore, the maximum number of active modes can be expected in each cross-section, orthogonality must be maintained between the modes in order to consider them as propagating channels. In addition, High antenna array size MIMO systems cannot be efficient in narrow tunnels, especially in the far-field region. If we want to extend this study a mode expectation analysis for a defined tunnel shape will be crucial to expect the number of MIMO antenna array. In addition, the antenna position must be taken under consideration as it can change the intensities of the excited modes at the transmitter. 


\section{References}

1. R. E. Collin, Field Theory of Guided Waves. New York: IEEE Press, 1991.

2. C. Briso-Rodriguez, J. M. Cruz, and J. I. Alonso, "Measurements and Modeling of Distributed Antenna Systems in Railway Tunnels," IEEE Transactions on Vehicular Technology, vol. 56, no. 5, pp. 2870-2879, 2007

3. K. D. Laakmann and W. H. Steier, "Waveguides: characteristic modes of hollow rectangular dielectric waveguides," Appl. Optics, vol. 15, no. 5, pp. 13341340, May 1976.

4. Sun, Z. and I. F. Akyildiz (2010). "Channel modeling and analysis for wireless networks in underground mines and road tunnels." IEEE Transactions on Communications 58(6): 1758-1768.

5. A. E. Forooshani, R. D. White, and D. G. Michelson, "Effect of antenna array properties on multiple-inputmultiple-output system performance in an underground mine," IET Microwaves, Antennas \& Propagation, vol. 7, no. 13, pp. 1035-1044, 2013.

6. D. Pozr, Microwave Engineering. New York: Wiley, 1998

7. S. F. Mahmoud and J. R. Wait, Guided electromagnetic waves in a curved rectangular mine tunnel vol. 9, 1974.

8. J. M. Molina-Garcia-Pardo, M. Lienard, P. Degauque, D. G. Dudley, and L. Juan-Llacer, "Interpretation of MIMO Channel Characteristics in Rectangular Tunnels From Modal Theory," IEEE Transactions on Vehicular Technology, vol. 57, no. 3, pp. 1974-1979, 2008.

9. S. Loyka, "Multiantenna capacities of waveguide and cavity channels," in CCECE 2003 - Canadian Conference on Electrical and Computer Engineering. Toward a Caring and Humane Technology (Cat. No.03CH37436), 2003, vol. 3, pp. 1509-1514 vol.3.

10. P. Delogne, Leaky Feeders and Subsurface Radio Communications. New York: Stevenage, Herts; P. Peregrinus, Aug. 1982

11. Forooshani, A. E., et al. (2013). "Effect of antenna array properties on multiple-input-multiple-output system performance in an underground mine." IET Microwaves, Antennas \& Propagation 7(13): 10351044.

12. M. Lienard, P. Degauque, J. Baudet, and D. Degardin, "Investigation on MIMO channels in subway tunnels," IEEE Journal on Selected Areas in Communications, vol. 21, no. 3, pp. 332-339, 2003. 